\title{
Ginsburg-Landau equation around the superconductor-insulator transition
}

\author{
T. K. Ng \\ AT\&T Bell Laboratories, Murray Hill, New Jersey 07974 \\ (Received 14 September 1990; revised manuscript received 28 December 1990)
}

\begin{abstract}
Based on the scaling theory of localization, we construct a Ginsburg-Landau (GL) equation for superconductors in an arbitrary strength of disordered potential. Using this GL equation, we reexamine the criteria for the superconductor-insulator transition and find that the transition to a localized superconductor can happen on both sides of the (normal) metal-insulator transition, in contrast to a previous prediction by Ma and Lee [Phys. Rev. B 32, 5658 (1985)] that the transition can only be on the insulator side. Furthermore, by comparing our theory with a recent scaling theory of dirty bosons by Fisher et al. [Phys. Rev. Lett. 64, 587 (1990)] we conclude that nontrivial crossover behavior in transport properties may occur in the vicinity of the superconductor-insulator transition.
\end{abstract}

In the past few years, there has been interest in the effect of disorder and localization in superconductors, both from the theoretical ${ }^{1-6}$ and experimental ${ }^{7,8}$ side. $\mathrm{Ma}$ and $\mathrm{Lee}^{1}$ have addressed the problem in the strongdisorder limit, where all (one-electron) states are localized. They concluded that superfluidity can be destroyed (at temperature $T=0$ ), only in the limit $\rho \Delta_{0} L^{d}<1$, where $\rho$ is the averaged density of states on the Fermi surface, $\Delta_{0}$ is the BCS gap, $L$ is the localization length, and $d$ is the dimension of the system. A similar conclusion was also reached by Kotliar and Kapitulnik. ${ }^{4,5}$ We shall address this problem again in this paper, starting from the weak-disorder limit where the effect of disorder will be reexamined using a Ginsburg-Landau (GL) equation with disorder properly included. Our result will then be extended to the scaling regime close to metal-insulator transition via the scaling approach of Kotliar and Kapitulnik, ${ }^{4,5}$ where the superconductor-insulator transition problem will be reexamined.

Following $\mathrm{Ma}$ and Lee, ${ }^{1}$ and Kotliar and Kapitulnik, ${ }^{4,5}$ we consider the generalized BCS Hamiltonian

$$
H=H_{0}-V \int \Psi_{\uparrow}^{\dagger}(\mathbf{r}) \Psi_{\downarrow}^{\dagger}(\mathbf{r}) \Psi \downarrow(\mathbf{r}) \Psi \uparrow(\mathbf{r}) d^{d} r,
$$

where $H_{0}$ is the single-particle part of the Hamiltonian describing noninteracting electrons moving in a disordered potential, and the second term is the usual BCS interaction.

In the Bogoliubov-de Gennes ${ }^{9}$ mean-field approximation, the superconductivity order parameter $\Delta(\mathbf{r})=V\langle\Psi \uparrow(\mathbf{r}) \Psi \downarrow(\mathbf{r})\rangle$ is determined by solving the following set of self-consistent equations:

$$
\begin{aligned}
& \varepsilon_{n} U_{n}(\mathbf{r})=H_{0} U_{n}(\mathbf{r})+\Delta(\mathbf{r}) V_{n}(\mathbf{r}), \\
& -\varepsilon_{n} V_{n}(\mathbf{r})=H_{0}^{*} V_{n}(\mathbf{r})-\Delta^{*}(\mathbf{r}) U_{n}(\mathbf{r}),
\end{aligned}
$$

and

$$
\Delta(\mathbf{r})=V \sum_{n} V_{n}^{*}(\mathbf{r}) U_{n}(\mathbf{r})\left[1-2 n_{F}\left(\varepsilon_{n}\right)\right],
$$

where $n_{F}(\varepsilon)$ is the Fermi-Dirac distribution function.

In the limit $\Delta(\mathbf{r})$ is small, one can expand Eq. (2) in powers of $\Delta(\mathbf{r})$. After some trivial algebra, we obtain the well-known result ${ }^{9,10}$

$$
\begin{aligned}
\Delta(\mathbf{r})= & \int K\left(\mathbf{r}, \mathbf{r}^{\prime}\right) \Delta\left(\mathbf{r}^{\prime}\right) d^{d} r^{\prime} \\
& +\int K_{4}\left(\mathbf{r}, \mathbf{r}^{\prime}, \mathbf{r}^{\prime \prime}, \mathbf{r}^{\prime \prime \prime}\right) \Delta\left(\mathbf{r}^{\prime}\right) \Delta^{*}\left(\mathbf{r}^{\prime \prime}\right) \Delta\left(\mathbf{r}^{\prime \prime \prime}\right) \\
& \times d^{d} \boldsymbol{r}^{\prime} d^{d} \boldsymbol{r}^{\prime \prime} d^{d} \boldsymbol{r}^{\prime \prime \prime}
\end{aligned}
$$

to third order in $\Delta(\mathbf{r})$, where

$$
\begin{gathered}
K\left(\mathbf{r}_{1}, \mathbf{r}_{2}\right)=\frac{V}{\beta} \sum_{i \omega_{n}} G\left(\mathbf{r}_{1}, \mathbf{r}_{2}, i \omega_{n}\right) G\left(\mathbf{r}_{1}, \mathbf{r}_{2},-i \omega_{n}\right), \\
K_{4}\left(\mathbf{r}_{1}, \mathbf{r}_{2}, \mathbf{r}_{3}, \mathbf{r}_{4}\right)=\frac{-V}{\beta} \sum_{i \omega_{n}} G\left(\mathbf{r}_{1}, \mathbf{r}_{2}, i \omega_{n}\right) G\left(\mathbf{r}_{1}, \mathbf{r}_{3},-i \omega_{n}\right) \\
\times G\left(\mathbf{r}_{4}, \mathbf{r}_{2}, i \omega_{n}\right) G\left(\mathbf{r}_{4}, \mathbf{r}_{3},-i \omega_{n}\right),
\end{gathered}
$$

and

$$
G\left(\mathbf{r}, \mathbf{r}^{\prime}, \omega\right)=\sum_{n} \frac{\Psi_{n}(\mathbf{r}) \Psi_{n}^{*}\left(\mathbf{r}^{\prime}\right)}{\omega-\varepsilon_{n}} .
$$

$G$ is the exact one-particle Green's function for an electron moving in the Hamiltonian $H_{0}$.

To derive the GL equation, we assume that $\Delta(\mathbf{r})$ is slowly varying within a distance of approximately the range of the kernals $K$ and $K_{4}$. Expanding

$$
\Delta\left(\mathbf{r}^{\prime}\right)=\Delta(\mathbf{r})+\left(\mathbf{r}^{\prime}-\mathbf{r}\right) \cdot \nabla \Delta(\mathbf{r})+\frac{1}{2}\left|\mathbf{r}-\mathbf{r}^{\prime}\right|^{2} \nabla^{2} \Delta(\mathbf{r})+\cdots
$$

and keeping leading order terms only, we obtain

$$
\begin{aligned}
\Delta(\mathbf{r})= & K_{0}(\mathbf{r}) \Delta(\mathbf{r})+\frac{1}{2} K_{1}(\mathbf{r}) \nabla^{2} \Delta(\mathbf{r}) \\
& +K_{2}(\mathbf{r}) \Delta(\mathbf{r})|\Delta(\mathbf{r})|^{2},
\end{aligned}
$$

where

$$
K_{0}(\mathbf{r})=\int K\left(\mathbf{r}, \mathbf{r}^{\prime}\right) d^{d} \boldsymbol{r}^{\prime}
$$

(c) 1991 The American Physical Society 


$$
K_{1}(\mathbf{r})=\int\left(\mathbf{r}-\mathbf{r}^{\prime}\right)^{2} K\left(\mathbf{r}, \mathbf{r}^{\prime}\right) d^{d} r^{\prime}
$$

and

$$
K_{2}(\mathbf{r})=\iiint K_{4}\left(\mathbf{r}, \mathbf{r}_{1}, \mathbf{r}_{2}, \mathbf{r}_{3}\right) d^{d} r, d^{d} r_{2} d^{d} r_{3} .
$$

Furthermore, we keep disorder explicitly only in $K_{0}(\mathbf{r})$, i.e., we let

$$
\begin{aligned}
& K_{1}(\mathbf{r}) \rightarrow\left\langle K_{1}(\mathbf{r})\right\rangle_{\mathrm{av}}=K_{1}, \\
& K_{2}(\mathbf{r}) \rightarrow\left\langle K_{2}(\mathbf{r})\right\rangle_{\mathrm{av}}=K_{2},
\end{aligned}
$$

and

$$
K_{0}(\mathbf{r})=\left\langle K_{0}(\mathbf{r})\right\rangle_{\mathrm{av}}+\delta K_{0}(\mathbf{r})=K_{0}+\delta K_{0}(\mathbf{r}),
$$

where $\langle K\rangle_{\text {av }}$ denotes the impurity ensembles average of $K$ and

$$
\delta K_{0}(\mathbf{r})=K_{0}(\mathbf{r})-\left\langle K_{0}(\mathbf{r})\right\rangle_{\mathrm{av}} .
$$

With these approximations, we arrive at the following GL equation,

$$
\begin{aligned}
\frac{1}{2} K_{1} \nabla^{2} \Delta(\mathbf{r})+\left(K_{0}-1\right) \Delta(\mathbf{r})+ & \delta K_{0}(\mathbf{r}) \Delta(\mathbf{r}) \\
& +K_{2}|\Delta(\mathbf{r})|^{2} \Delta(\mathbf{r})=0,
\end{aligned}
$$

where the disorder potential $\delta K_{0}(\mathbf{r})$ now describes explicitly the effect of disorder on the order parameter $\Delta(\mathbf{r})$ (or the Cooper pairs), with all the effects of disorder on the one-particle eigenstates incorporated in the internal structure of the parameters' $K$ 's.

It is important to keep in mind that, in general, higher order terms appear in the GL equation, and the coefficients' $K$ 's are, in general, all random. Equation (9) is valid only when all of the important physics are lying in the infrared (long-wavelength) region, where only the terms present in Eq. (9) are "relevant."

The coefficients $K_{0}, K_{1}$, and $K_{2}$ were computed in the weak-disorder limit ${ }^{11}$ and were also being studied near the metal-insulator transition ${ }^{4,5}$ using scaling theory. We shall not repeat these calculations here but shall just state the results. In the weak-disorder limit it can be shown that $K_{0}$ and $K_{2}$ are disorder insensitive and are given by $^{4,5}$

$$
\begin{aligned}
& K_{0} \sim V \rho \ln \frac{\omega_{D}}{T}, \\
& K_{2} \sim \frac{-V \rho}{T_{c}^{2}},
\end{aligned}
$$

for temperature $T \sim$ critical temperature $T_{c} \cdot \rho$ is the average density of state on the Fermi surface, and $\omega_{D}$ is the Debye cutoff for the BCS interaction,

$$
K_{1} \sim \begin{cases}-V \rho \frac{v_{F}^{2}}{T_{c}^{2}} & \frac{1}{\tau} \ll T_{c} \\ -V \rho \frac{D}{T_{c}} & \frac{1}{\tau} \gg T_{c},\end{cases}
$$

where $D \sim v_{F} l$ is the diffusion coefficient, $v_{F}$ is the Fermi velocity, $l$ is the elastic mean-free path, and $\tau$ is the elastic-scattering time. The calculation can be extended to the scaling regime around the metal-insulator transition by considering a scale-dependent diffusion coefficient $D(q, \omega) .^{4,5}$

Notice that the zero-temperature coherence length $\xi_{0}$ is given by

$$
\xi_{0} \sim\left(\frac{-K_{1}}{K_{0}}\right)^{1 / 2}
$$

where it is easy to see from Eq. (10) that the coherence length is reduced by disorder to $\xi \sim\left(\xi_{\text {clean }} l\right)^{1 / 2}$ in the limit $1 / \tau>T_{c}$ (or $\xi_{\text {clean }}>l$ ).

Using Eqs. (3), (4), and (8), we find that the random potential $\delta K_{0}(\mathbf{r})$ is given by

$\delta K_{0}(\mathbf{r})=V \int_{-\omega_{D}}^{\omega_{D}} d \varepsilon \frac{1-2 n_{F}(\varepsilon)}{\epsilon}\left[N(\varepsilon, \mathbf{r})-\langle N(\varepsilon, \mathbf{r})\rangle_{\mathrm{av}}\right]$

where

$$
N(\varepsilon, \mathbf{r})=\sum_{\epsilon_{n}} \delta\left(\varepsilon-\varepsilon_{n}\right)\left|\Psi_{n}(\mathbf{r})\right|^{2}
$$

is the local density of state at point $\mathbf{r}$. However, we must keep in mind that, in our GL formulation, we have assumed that the order parameter is slowly varying within a length scale $\sim \xi_{0}$ (coherence length), and the GL equation is derived by assuming that all shorter length scale fluctuations are unimportant. Thus, to be consistent, the random potential $\delta K_{0}(\mathbf{r})$ that enters into the GL equation should describe only fluctuations in a length scale $\gtrsim \xi_{0}$, i.e., the "correct" random potential should be $\delta K_{0}(\mathbf{r})$ given in Eq. (2) averaged over a region of size $\xi_{0}^{d,}$, i.e.,

$$
\delta K_{0}^{c}(\mathbf{r})=\frac{1}{\Omega} \sum_{|q|<\xi_{0}^{-1}} \delta K_{0}(\mathbf{q}) e^{\mathrm{iq} \cdot \mathbf{r}}
$$

where $\delta K_{0}(\mathbf{q})$ is the Fourier transform of Eq. (12).

The disorder potential $\delta K_{0}(\mathbf{r})$ is characterized by its correlation function

$$
\left\langle\delta K_{0}(\mathbf{r}) \delta K_{0}\left(\mathbf{r}^{\prime}\right)\right\rangle_{\mathrm{av}}=V^{2} \int_{-\omega_{D}}^{\omega_{D}} d \varepsilon \int_{-\omega_{D}}^{\omega_{D}} d \varepsilon^{\prime} \frac{1-2 n_{F}(\varepsilon)}{\varepsilon} \frac{1-2 n_{F}\left(\varepsilon^{\prime}\right)}{\varepsilon^{\prime}}\left\langle\delta N(\varepsilon, \mathbf{r}) \delta N\left(\varepsilon^{\prime}, \mathbf{r}^{\prime}\right)\right\rangle_{\mathrm{av}},
$$

where $\delta N=N-\langle N\rangle_{\mathrm{av}}$. Thus $\delta K_{0}(\mathbf{r})$ is a direct measure of the fluctuation in local density of states $N(\varepsilon, \mathbf{r})$ of the dirty system.

The correlation function $\left\langle\delta N(\varepsilon, \mathbf{r}) \delta N\left(\varepsilon^{\prime}, \mathbf{r}^{\prime}\right)\right\rangle$ has been computed by Al'tshuler and Shklovskii ${ }^{13}$ in the weakdisorder limit, where they found that

$\left\langle\delta N(\varepsilon, \mathbf{r}) \delta N\left(\varepsilon^{\prime}, \mathbf{r}^{\prime}\right)\right\rangle_{\mathrm{av}} \simeq \frac{4}{\pi^{2}} \operatorname{Re}\left[\boldsymbol{P}\left(\mathbf{r}-\mathbf{r}^{\prime}, \varepsilon-\varepsilon^{\prime}\right)\right]^{2}$ 
for $\left|\mathbf{r}-\mathbf{r}^{\prime}\right|>l$ and $\varepsilon-\varepsilon^{\prime}<<1 / \tau$, where $P$ describes a diffusion or a Cooper propagator ${ }^{13,14}$ with Fourier transform

$$
P(\mathbf{q}, \omega)=\frac{1}{-i \omega+D q^{2}+\gamma},
$$

where $\gamma$ is the lifetime of the electron due to inelastic process (we assume $\gamma \ll T_{c}$ ), and $D$ is the diffusion constant.

Now let us consider first the limit $1 / \tau \gg T_{c}$. With Eqs. (13)-(16), we found that

$$
\begin{aligned}
\left\langle\delta K_{0}^{c}(\mathbf{r}) \delta K_{0}^{c}\left(\mathbf{r}^{\prime}\right)\right\rangle_{\mathrm{av}} \simeq \int_{-\omega_{D}}^{\omega_{D}} d \varepsilon \frac{1-2 n_{F}(\varepsilon)}{\varepsilon} & \\
& \times \int_{-\omega_{D}}^{\omega_{D}} d \varepsilon \frac{1-2 n_{F}\left(\varepsilon^{\prime}\right)}{\varepsilon^{\prime}} \frac{4}{\pi^{2}} \operatorname{Re} \frac{1}{\Omega^{2}} \\
& \quad \times \int_{|\mathbf{q}|<\xi_{0}^{-1}} d^{d} q \int_{\left|\mathbf{q}^{\prime}\right|<\xi_{0}^{-1}} d^{d} q^{\prime} P\left(q, \varepsilon-\varepsilon^{\prime}\right) P\left(q^{\prime}, \varepsilon-\varepsilon^{\prime}\right) e^{i\left(\mathbf{q}+\mathbf{q}^{\prime}\right) \cdot\left(\mathbf{r}-\mathbf{r}^{\prime}\right)}
\end{aligned}
$$

Notice that $D q^{2} \lesssim D \xi_{0}^{-2} \sim T_{c}$ in the limit $(1 / \tau) \gg T_{c}$, so that $P(q, \varepsilon) \sim(-i \varepsilon+\gamma)^{-1}$ for $\varepsilon \gtrsim T_{c}$. With this, the integral in Eq. (17) can be estimated, giving

$$
\left\langle\delta K_{0}^{c}(\mathbf{r}) \delta K_{0}^{c}\left(\mathbf{r}^{\prime}\right)\right\rangle \sim \frac{V^{2}}{\left(\xi_{0}\right)^{2 d}} \frac{\delta^{d}\left(\mathbf{r}-\mathbf{r}^{\prime}\right)}{T_{c}^{2}} .
$$

In the other limit $1 / \tau<<T_{c}, \xi_{0} \ll<$ so that we are probing regions where electron motions are ballistic. $\left\langle N(\varepsilon, \mathbf{r}) N\left(\varepsilon^{\prime}, \mathbf{r}^{\prime}\right)\right\rangle$ becomes highly nonlocal because of the strong correlation over distance scale of length $l$, and so does the correlation function $\left\langle\delta K^{c} \delta K^{c}\right\rangle$.

However, in this situation, randomness comes in only for distance scale $\gtrsim l . \Delta(\mathbf{r})$ is expected to be smoothly varying over distances $\lesssim l$, so that information on distance scale $\sim \xi_{0}$ is not expected to be important. The interesting physics come in on scale $\gtrsim l$, so that it is more convenient to change the momentum cutoff in the GL equation to $l^{-1}$. With this replacement in Eq. (17), we find

$$
\left\langle\delta K_{0}^{c}(\mathbf{r}) \delta K_{0}^{c}\left(\mathbf{r}^{\prime}\right)\right\rangle \sim \frac{V^{2}}{l^{2 d}} \frac{\delta^{d}\left(\mathbf{r}-\mathbf{r}^{\prime}\right)}{T_{c}^{2}} .
$$

Notice the change of the scale in the denominator from $\xi_{0}$ to $l$ in Eq. (19).

The physical meaning of Eqs. (18) and (19) is as follows: in the limit $\xi_{0}>l$, every region of size $\xi_{0}^{d}$ experiences a completely different (random) potential and, thus, a different (random) density of states. Therefore, $\delta K_{0}^{c}(\mathbf{r})$ is uncorrelated for distance $\left|\mathbf{r}-\mathbf{r}^{\prime}\right| \gtrsim \xi_{0}$, which results in the $\delta$ function in Eq. (18). The $\left(\xi_{0}\right)^{-2 d}$ term is a direct consequence of averaging over region of size $\xi_{0}^{d}$, whereas the $T_{c}^{-2}$ term comes from the enhanced correlation because of diffusive motion of electrons. ${ }^{14}$ In the limit $\xi_{0}<l, \xi_{0}$ is replaced by $l$, because only regions of size $\gtrsim l$ see truly "random" potential, which gives Eq. (19).

We shall now extend our result to the scaling regime near (normal) metal-insulator transition, following the approach of Kotliar and Kapitulnik, ${ }^{4,5}$ where the coefficients $K_{0}, K_{1}$, and $K_{2}$ were computed by considering a scale-dependent diffusion constant. They found that only $K_{1}$ changes in the scaling regime and their result can be summarized as the change in the zero- temperature coherence length $\xi_{0}$ as follows.

For $d>2$, above the mobility edge (i.e., metal side),

$$
\xi_{0}^{2} \sim\left\{\begin{array}{l}
l_{T_{c}}^{d} L^{-(d-2)}, \quad l_{T_{c}}>L \\
l_{T_{c}}^{2}, \quad l_{T_{c}}<L,
\end{array}\right.
$$

and below the mobility edge (insulator side),

$$
\xi_{0}^{2} \sim\left\{\begin{array}{l}
l_{T_{c}}^{2} \quad L_{T_{c}}<L \\
\ln \left(\frac{L_{T_{c}}}{L}\right)^{d} L^{2} \quad l_{T_{c}}>L,
\end{array}\right.
$$

where $l_{T}$ is the thermal length $l_{T}^{d} \sim(T \rho)^{-1}$, and $L$ is the localization length. (In the metal side, $L$ denotes the length scale above which the conductivity recovers the useful Drude character. ${ }^{14}$ )

These results can be interpreted in the following way. ${ }^{5}$ Let us consider Eq. (11), where the coherence length is given by

$$
\xi_{0}=\left(\xi_{\text {clean }} \frac{D}{v_{F}}\right)^{1 / 2} .
$$

As we approach the mobility edge, the expression $D \sim v_{F} l$ ceases to be valid, and should be replaced by the scaling form $D \sim\left(\rho L^{d-2}\right)^{-1} \cdot{ }^{14}$ Making this replacement in Eq. (22) gives Eq. (20a). As we get closer to the mobility edge, $L$ increases and $\xi_{0}$ decreases until they cross at $L \sim \xi_{0} \sim l_{T_{c}}$. Physically, when the localization length is larger than the coherence length $\xi_{0}$, the Cooper pair cannot distinguish between metal and insulator. Thus, when $\xi_{0}<L$, the zero-temperature coherence length cannot depend on $L$ and is, therefore, saturated at $\xi_{0} \sim l_{T_{c}}$ [Eq. (20b)]. As one crosses over to the insulator side, again, for $L>l_{T_{c}}$, the Cooper pair cannot distinguish between metal and insulator and thus, $\xi_{0}$ is still saturated at $l_{T_{c}}$. It is only when $L<l_{T_{c}}$ that the coherence length $\xi_{0}$ 
starts to decrease again. Notice that, in this regime, the size of the one-electron wave function is of order $L$, therefore, we expect $\xi_{0} \sim L$ because the size of the Cooper pairs should not be larger than the size of a localized one-electron wave function.

In $2 \mathrm{D}$, when all states are localized, we can repeat similar analysis with the following result for $\xi_{0}$,

$$
\xi_{0}\left\{\begin{array}{cl}
\sim \bar{\xi}_{0} & L \gg \bar{\xi}_{0} \\
\sim L & L<\bar{\xi}_{0},
\end{array}\right.
$$

where $L \sim l e^{(\pi / 2)\left(k_{F} l\right)}$ is the localization length in two dimension and $\bar{\xi}_{0} \sim\left(\xi_{\text {clean }} l\right)^{1 / 2}$. Notice that the thermal length does not enter because $\xi_{0} \gg l_{T}\left(T \sim T_{c}\right)$ in 2D whenever $L \gtrsim \bar{\xi}_{0}$. For $L<\bar{\xi}_{0}, \xi_{0}$ is again cutoff by $L$ for the same reason as in the $3 \mathrm{D}$ case.

The calculation of $\left\langle\delta K_{0}^{c}(\mathbf{r}) \delta K_{0}^{c}\left(\mathbf{r}^{\prime}\right)\right\rangle$ can also be extended to the scaling regime by replacing $D$ with $D(q, \omega)$ in Eq. (16). It can easily be seen that the same expression Eq. (18) is obtained [except that $\xi_{0}$ is replaced by the scaling form Eqs. (20)-(24)]. The reason is that, for $q<\xi_{0}^{-1}$, $D q^{2}<T_{c}$ from Eq. (22), so that $D q^{2}$ term is never important in determining $\left\langle\delta K_{0}^{c}(\mathbf{r}) \delta K_{0}^{c}\left(\mathbf{r}^{\prime}\right)\right\rangle$. Physically, the correlation between density of states is a local property of the system and is not expected to be affected by localization effects around the mobility edge that are long-range in character. Our analysis is expected to break down in the insulating side only when $\rho T_{c} L^{d} \lesssim 1$, when the average number of states within a volume $L^{d}$ with energy $|\varepsilon|<T_{c}$ is so small that fluctuation in the number of states become dominating.

Now we shall consider some consequences of our GL equation. Kotliar and Kapitulnik ${ }^{4,5}$ have considered a similar GL equation except that they have neglected the "disorder potential" $\delta K_{0}^{c}(\mathbf{r})$ so that the effect of disorder comes in only through the decrease in $\xi_{0}$. We shall show that although the $\delta K_{0}^{c}(\mathbf{r})$ term is indeed unimportant in the weak-disorder limit, it becomes important as the system approaches metal-insulator transition and leads to a new prediction about the superconductor-insulator transition.

Before presenting our analysis, we shall first say a few words about the meaning of our GL equation around the superconductor-insulator transition. Ma et al. ${ }^{1,3}$ showed that a superconductor-insulator transition does not occur in a mean-field theory of dirty bosons, and the actual (zero-temperature) superconductor-insulator transition is driven by quantum fluctuation, which destroys the longranged phase coherence of the boson field. Thus, strictly speaking, a GL equation alone cannot describe a superconductor-insulator transition. The GL equation we derived should instead be understood as giving an effective Ginsburg-Landau energy functional

$$
\begin{aligned}
F[\Psi]=\int\{ & K_{1}|\nabla \Psi|^{2}+K_{2}\left[|\Psi|^{2}-\Delta(T)\right]^{2} \\
& \left.+K_{t}\left|\partial_{t} \Psi\right|^{2}+\delta K_{0}^{c}(r)|\Psi|^{2}\right\} d^{d} x d t,
\end{aligned}
$$

and the partition functional of the physical system is given by the path integral over all possible boson field configurations $\Psi$ weighted by $\exp [-F[\Psi]]$. (Notice that a time derivative term should also be present in $F[\Psi]$ in order to describe quantum fluctuations correctly.) Assuming an average density of state $\langle N(\varepsilon)\rangle_{\mathrm{av}}$, which is symmetric around the Fermi surface, it is easy to obtain the time derivative term with $K_{t}$ of order $V \rho T_{c}^{-2}$.

In the renormalization group (RG) sense, the GL functional $F[\Psi]$ should be considered as giving a renormalized Hamiltonian for the system that is valid at energy scale $\lesssim T_{c}$, where fermionic excitations are suppressed by the superconducting gap, and the relevant degrees of freedom are the fluctuations of the boson field of Cooper pairs.

To describe physical processes at energy scale $\ll T_{c}$ (for example, the zero-temperature superconductorinsulator transition), quantum fluctuations of the boson field must be taken into account that would further renormalize the effective GL functional $F[\Psi]$. These kinds of renormalization effects have been discussed by several authors, ${ }^{2,3,6}$ and we shall not go into detail here. We shall just ask one question in the following, at what strength of disorder is a superconductor-insulator transition going to occur?

Notice that although the detail behavior of the system around the superconductor-insulator transition can only be obtained after an extensive analysis of the path integral, the approximate position of the (zero-temperature) transition can be read off from the "bare" GL energy functional $F[\Psi]$, where it is believed that a transition is going to occur when the ratio between the kinetic energy $W$ of the system and the strength of disorder potential $\left|\delta K_{0}^{c}(r)\right|$ is of order 1 , at least for dimension $d>1 ., 3,6$ Physically, the situation is very similar to the fermion localization problem at $d>2$, where it is known that the (zero-temperature) metal-insulator transition occurs when $W /\left|\delta K_{0}^{c}\right| \sim \mathrm{O}(1)$, but the detail behavior of the transition can be obtained only after an extensive RG analysis. $^{14}$

In the weak-disorder limit, the magnitude of the disorder potential $\delta K_{0}^{c}(\mathbf{r})$ is given by (in the limit $\xi_{0} \gg l$ ) $\left|\delta K_{0}^{c}\right| \sim V /\left(\xi_{0}^{d} T_{c}\right)$, whereas the kinetic energy in the GL equation is of order $\sim K_{1} / \xi_{0}^{2} \sim V \rho$. With $\xi_{0} \sim D / T_{c}$, we find $\left|\delta K_{0}\right| /\left(\right.$ kinetic energy) $\sim\left(k_{F} l\right)^{-1}<<1$ in the weakdisorder limit, showing that the disorder potential $\delta K_{0}^{c}(\mathbf{r})$ is indeed negligible.

To examine the scaling regime, first we observe that the ratio $\alpha$ between magnitudes of $\delta K_{0}^{c}(\mathbf{r})$ and kinetic energy can be written in general as

$$
\alpha \sim \frac{1}{\xi_{0}^{d} T_{c} \rho},
$$

and the disorder potential will be important and can lead to a superconductor-insulator transition when $\alpha \gtrsim 1$, or $\xi_{0}^{d} T_{c} \rho \lesssim 1$. This inequality has a simple physical meaning. Notice that the left-hand side of the inequality is the average number of states in a spacial region of volume $\xi_{0}^{d}$ located at an energy range $|\varepsilon|<T_{c}$, i.e., the number of electronic states involved in forming a Cooper pair. Thus, the inequality has the simple physical interpretation that the effects of electronic disorder become impor- 
tant when the average number of electronic states involved in forming one Cooper pair is of order 1, so that fluctuation in density of states become dominating. ${ }^{1,13}$ Notice that based on a physical argument, $\mathrm{Ma}$ and $\mathrm{Lee}^{1}$ have arrived at a similar criteria for superconductorinsulator transition, except that they have replaced $\xi_{0}$ by the localization length $L$ on the insulator side, i.e., they assumed that the superconductor-insulator transition always takes place on the insulator side of the (normal) metal-insulator transition [see Eqs. (20)-(23)].

In 2D, with Eqs. (23) and (24), we find that the strength of disorder potential $\delta K_{0}^{c}(\mathbf{r})$ increases continuously with increasing disorder, and is comparable with the kinetic energy when $\xi_{0} \sim L \sim l_{T_{c}}$, where a superconductorinsulator transition is expected to take place in agreement with the conclusion of Ma and Lee. ${ }^{1}$

In 3D, however, with Eqs. (20)-(22), we find that the effective strength of $\delta K_{0}^{c}(\mathbf{r})$ does not increase continuously with increasing disorder. There is a region around the metal-insulator transition when $L>l_{T_{c}}$ where the coherence length $\xi_{0}$ becomes disorder independent $\left(\xi_{0} \sim l_{T_{c}}\right)$. With Eq. (24), we find that the strength of $\delta K_{0}^{c}(\mathbf{r})$ is comparable with the kinetic energy precisely at this region, where the superconductor-insulator transition is expected to take place. However, since this region covers both the metal and the insulator side of the metal-insulator transition, we conclude that it is possible to have a superconductor-insulator transition both above and below the metal-insulator transition, in contrast to the prediction of $\mathrm{Ma}$ and Lee. ${ }^{1,3}$ The exact position of transition depends on the microscopic details of the system and is not obtainable from our "average" theory. Notice, however, that although the exact position of transition is nonuniversal, there may exist other properties that are "universal" around the transition. In fact, starting from a dirty-boson theory, Fisher, Grinstein, and Girvin ${ }^{2}$ proposed that the transport coefficients may have universal behavior around the transition as temperature $T \rightarrow 0$, where

$$
\left\{\begin{array}{l}
G \rightarrow C_{0} \frac{e^{2}}{h} \quad(2 \mathrm{D}) \\
\sigma \propto T(3 \mathrm{D}),
\end{array}\right.
$$

where $G$ is the conductance at $2 \mathrm{D}$, and $C_{0}$ is a universal number. $\sigma$ is the $3 \mathrm{D}$ conductivity.

Their result is applicable when $T \ll T_{c}$, where $T_{c}$ is the temperature for formation of Cooper pairs, not the temperature for onset of superfluidity. In this regime, the "quasiparticle" of the system are bosons (Cooper pairs) and the problem can be treated as a dirty-boson problem. To complete the study, we shall consider transport behavior at $T>T_{c}$, where the "quasi-particles" are still fermions, and we shall compare experimental predictions at these two different temperature regimes.

In $2 \mathrm{D}$, scaling theory predicts that the conductance of a fermionic gas is given by

$$
G \sim \frac{e^{2}}{h} e^{-l_{\text {in }}(T) / L},
$$

where $l_{\text {in }}(T)$ is the inelastic mean-free path, which we have assumed $l_{\text {in }}(T)>L$. Around the superconductorinsulator transition. $L \sim l_{T_{c}} \sim \xi_{0}$ [Eq. (24)], thus, we have

$$
G \sim \frac{e^{2}}{h} e^{-l_{\text {in }}(T) / l_{T}} \lesssim \frac{e^{2}}{h},
$$

where we have assumed $l_{\text {in }}(T)>\xi_{0}\left(=l_{T_{c}}\right)$. This assumption is valid for most inelastic scattering mechanisms (phonons, $e-e$ scattering) at low temperature. In fact, when $l_{\text {in }}(T)<\xi_{0}$, the Cooper pairs would have already decayed before they propagate a distance $\xi_{0}$, and one expects that "true" superconductivity can occur only when $\xi_{0} \lesssim l_{\text {in }}(T),{ }^{15}$ when the inequality (27) is valid.

In $3 \mathrm{D}$, on the metal side of the metal-insulator transition, the conductivity $\sigma$ is given by

$$
\sigma \sim \frac{e^{2}}{h L} \quad L<l_{\text {in }}(T) .
$$

Around the superconductor-insulator transition, we have $L \gtrsim l_{T_{c}}$, so that with $l_{\text {in }}(T)>l_{T_{c}}$, we have

$$
\sigma \lesssim \frac{e^{2}}{h l_{T_{c}}} \sim \frac{e^{2}}{h}\left(\rho T_{c}\right)^{1 / 3},
$$

for the dc conductivity.

It is obvious that our results, which are nonuniversal (the transport coefficients are bound by inequality only), do not extrapolate to the "boson" results proposed by Fisher et $a l .^{2}$ smoothly, indicating that there may exist a crossover regime $0 \lesssim T \lesssim T_{c}$ where the transport behavior changes nonuniversally.

However, one has to keep in mind that the scaling theory we used is straightly speaking applicable only for noninteracting electrons, and it is possible that the theoretical predictions are strongly renormalized due to the BCS attractive interaction between electrons around $T_{c}$. In fact, a rough estimation of $l_{\text {in }}(T)$ from electron scattering from virtual Cooper pairs at $T \gtrsim T_{c}$ suggests that $l_{\text {in }}(T) \rightarrow l_{T}$ in the regime around superconductorinsulator transition, when $\xi_{0} \rightarrow l_{T}$, indicating that the scaling predictions are strongly renormalized and a "smooth" interpolation between the boson and fermion regimes may be possible. (Notice that it is also likely that the zero-temperature theory of Fisher et al. ${ }^{2}$ be strongly renormalized around $T_{c}$ due to thermal fluctuations.) It would be interesting to determine the transport behavior in this crossover regime experimentally.

In conclusion, using standard perturbation techniques and scaling theory for noninteracting electrons, we construct a GL equation for dirty superconductors that is valid for both strong and weak disorder. We reexamine the superconductor-insulator transition and find that the transition may occur at either side of the metal-insulator transition, depending on microscopic details. We also study the transport behavior around the superconductor-insulator transition, and find that nonuniversal crossover behavior was found around the BCS transition temperature $T_{c}$ suggesting that existing 
theories have to be strongly renormalized around this temperature regime. Further experimental and theoretical works are needed to understand this regime where fermion transport is crossed over to boson transport.
The author thanks C. M. Varma for directing him to the dirty-superconductor problem and for useful discussions and also P. A. Lee and R. N. Bhatt for useful discussion throughout the work.
${ }^{1}$ M. Ma and P. A. Lee, Phys. Rev. B 32, 5658 (1985).

${ }^{2}$ M. P. A. Fisher, G. Grinstein, and S. M. Girvin, Phys. Rev. Lett. 64, 587 (1990).

${ }^{3}$ M. Ma, B. I. Halperin, and P. A. Lee, Phys. Rev. B 34, 3136 (1986).

${ }^{4}$ A. Kapitulnik and G. Kotliar, Phys. Rev. Lett. 54, 473 (1985).

${ }^{5}$ A. Kapitulnik and G. Kotliar, Phys. Rev. B 33, 3146 (1986).

${ }^{6}$ M. P. A. Fisher, P. B. Weichman, G. Grinstein, and D. S. Fisher, Phys. Rev. B 40, 546 (1989).

${ }^{7}$ D. B. Haviland, Y. Liu, and A. M. Goldman, Phys. Rev. Lett. 62, 2180 (1989).

${ }^{8}$ H. M. Jaeger, D. B. Haviland, B. G. Orr, and A. M. Goldman, Phys. Rev. B 40, 182 (1989).

${ }^{9}$ See, for example, P. D. de Gennes, Superconductivity of Metals and Alloys (Benjamin, New York, 1966).

${ }^{10}$ See, for example, J. R. Schrieffer, Theory of Superconductivity (Benjamin/Cummings, New York, 1964).

${ }^{11}$ A. Abrikosov and L. P. Gorkov, Zh. Eksp. Teor. Fiz. 35, 1558 (1989) [Sov. Phys. JETP 8, 1090 (1959)]; 36, 319 (1959) [9, 220, (1959)].

${ }^{12}$ See, for example, G. Parisi, Statistical Field Theory (AddisonWesley, Reading, MA, 1988), for a description of scaling.

${ }^{13}$ B. L. Al'tshuler and E. I. Shklovskii, Zh. Eksp. Teor. Fiz. 91, 220 (1986) [Sov. Phys. JETP 64, 127 (1986)].

${ }^{14}$ See, for example, P. A. Lee and T. V. Ramakrishnan, Rev. Mod. Phys. 57, 287 (1985).

${ }^{15}$ P. A. Lee and N. Read, Phys. Rev. Lett. 58, 2691 (1987). 